\title{
Identification of small RNAs in Francisella tularensis
}

\author{
Guillaume Postic ${ }^{1,2}$, Eric Frapy ${ }^{1,2}$, Marion Dupuis ${ }^{1,2}$, Iharilalao Dubail ${ }^{1,2}$, Jonathan Livny ${ }^{3,4}$, Alain Charbit ${ }^{1,2}$, \\ Karin L Meibom ${ }^{1,2^{*}}$
}

\begin{abstract}
Background: Regulation of bacterial gene expression by small RNAs (sRNAs) have proved to be important for many biological processes. Francisella tularensis is a highly pathogenic Gram-negative bacterium that causes the disease tularaemia in humans and animals. Relatively little is known about the regulatory networks existing in this organism that allows it to survive in a wide array of environments and no sRNA regulators have been identified so far.

Results: We have used a combination of experimental assays and in silico prediction to identify sRNAs in F. tularensis strain LVS. Using a CDNA cloning and sequencing approach we have shown that $F$. tularensis expresses homologues of several sRNAs that are well-conserved among diverse bacteria. We have also discovered two abundant putative sRNAs that share no sequence similarity or conserved genomic context with any previously annotated regulatory transcripts. Deletion of either of these two loci led to significant changes in the expression of several mRNAs that likely include the cognate target(s) of these sRNAs. Deletion of these sRNAs did not, however, significantly alter $F$. tularensis growth under various stress conditions in vitro, its replication in murine cells, or its ability to induce disease in a mouse model of $F$. tularensis infection. We also conducted a genome-wide in silico search for intergenic loci that suggests $F$. tularensis encodes several other sRNAs in addition to the sRNAs found in our experimental screen.
\end{abstract}

Conclusion: Our findings suggest that $F$. tularensis encodes a significant number of non-coding regulatory RNAs, including members of well conserved families of structural and housekeeping RNAs and other poorly conserved transcripts that may have evolved more recently to help F. tularensis deal with the unique and diverse set of environments with which it must contend.

\section{Background}

RNA regulators are important players in control of gene expression in bacteria and often mediate a response to changes in the environment (for review, see [1]). Some regulatory RNAs, designated riboswitches, are part of the mRNA they regulate. Riboswitches are sequences in the 5 ' end of mRNAs that change conformation upon binding of a ligand, affecting transcription or translation of the down-stream gene (positively or negatively). Other RNA regulators bind to proteins and regulate their function, whereas the largest group of small RNAs (sRNAs), act by base pairing with target RNAs. Base pairing sRNAs generally fall into two groups: cis-acting sRNAs that have capacity for extensive base pairing, and trans-

\footnotetext{
* Correspondence: karin.meibom@inserm.fr

${ }^{1}$ INSERM U1002, Paris, France

Full list of author information is available at the end of the article
}

encoded sRNAs with a more limited potential for base pairing with their target RNA. Trans-acting sRNAs regulate the translation and/or the stability of their target RNAs and each often regulate more than one target. For the most part, these sRNAs affect target genes in a negative fashion by binding to the region surrounding the start codon and ribosome binding site, but can act through base pairing in a region far upstream, and occasionally affect translation positively (for review see [1]). Many of the trans-encoded sRNAs require the RNA chaperone Hfq for function. Hfq promotes RNA-RNA interactions between the sRNA and its target mRNA and the protein may additionally stabilize the sRNA in vivo.

RNA regulators furthermore control pathogenesis in bacteria such as Staphylococcus aureus [2-6], Salmonella typhimurium [7-10], Vibrio cholerae [11-13], group A Streptococcus [14,15], Pseudomonas aeruginosa [16-19],
Ciomed Central

C 2010 Postic et al; licensee BioMed Central Ltd. This is an Open Access article distributed under the terms of the Creative Commons Attribution License (http://creativecommons.org/licenses/by/2.0), which permits unrestricted use, distribution, and reproduction in any medium, provided the original work is properly cited. 
Clamydia trachomatis [20], and Clostridium perfringens [21,22].

Francisella tularensis is a Gram-negative bacterium that causes the disease tularaemia in humans and in a large number of animals and is one of the most virulent bacterial pathogens known. The ability of $F$. tularensis to invade and replicate in host cells, particularly in immune cells such as macrophages, is critical for its capacity to cause disease. Once inside host cells, $F$. tularensis resides transiently inside a phagosome that matures into a late endosomal stage and the bacterium then escapes to replicate in the cytoplasm. The mechanism by which the bacterium escapes the phagosome is not well understood, but genes encoded in a pathogenicity island (FPI) are required for this step in the infectious cycle. Several regulatory proteins regulate virulence gene expression in F. tularensis by activating transcription of the FPI. These comprise the proteins MglA [23,24], SspA [25], PrmA [26], and FevR (or PigR) [27,28]. F. tularensis unlike many other bacteria encodes only one alternative sigma factor [29], no complete two component regulator pairs [26], and very little information exists concerning control of gene expression apart from the above mentioned regulation of the FPI.

We have recently studied the role of Hfq in the physiology and virulence of $F$. tularensis [30]. Transcriptional analyses revealed that Hfq - directly or indirectly - regulates the expression of numerous genes in this pathogen. Functional studies showed that Hfq is required for stress resistance as well as for full virulence in both a fully virulent strain and in the attenuated live vaccine strain (LVS). Since Hfq normally performs its function by promoting sRNA-mRNA interactions, these results strongly suggest that sRNAs are expressed in this organism and are involved in diverse functions. Here, we have initiated the identification of the sRNAs expressed by $F$. tularensis LVS. We have assessed and identified the sRNA species that are expressed at high levels and found the commonly known 4.5S RNA, 5S rRNA, and the transfer messenger RNA (tmRNA). Additionally, we identified two novel sRNA species by cDNA cloning and have characterized these. Studies of sRNA mutant strains suggested potential targets for regulation. As an alternative approach to identify sRNAs in $F$. tularensis, we performed a bioinformatic prediction of sRNAs. Interestingly, this analysis found both the experimentally determined sRNA loci and additionally identified a number of other putative sRNA-containing intergenic regions.

\section{Results and Discussion}

Identification of highly expressed small RNA species of Francisella

To identify sRNAs in $F$. tularensis we first assessed the highly expressed sRNAs by polyacrylamide gel electrophoresis followed by ethidium bromide staining (Figure 1). This approach has been used successfully to find novel sRNAs in S. aureus [31]. We isolated total RNA from $F$. tularensis LVS after growth in normal complex broth in exponential growth phase (E) or in stationary phase $(\mathrm{S})$, after oxidative stress $(10 \mathrm{mM}$ $\mathrm{H}_{2} \mathrm{O}_{2}$ ) or after growth at high osmolarity (Schaedler broth $+2 \% \mathrm{NaCl}$ ). We chose to assess the RNA profile under different conditions because sRNA expression in other bacteria was found to be constitutive for some sRNAs (mostly cis-encoded sRNAs), but induced under specific conditions (such as exposure to stress) for most trans-encoded sRNAs [1]. However, no major differences were observed in the pattern of RNA species that were visible after PAGE (Figure 1A). Four bands ranging in size from $\sim 100$ nt to $\sim 400$ nt were visible after staining (Figure 1A; labeled 1-4). To determine the origin of these four RNA species, we eluted the RNAs from the gel and performed linker ligation, cDNA synthesis, and PCR amplification of each eluted RNA (see Methods). Sequence analysis of cloned PCR fragments identified the RNAs, although in all cases we also found contaminating rRNA and/or tRNA sequences.

Ten clones corresponding to RNA \#4, the largest RNA, were sequenced and seven of these identified the RNA as the transfer messenger RNA (tmRNA; FTL_R001). This RNA is also designated SsrA and is $421 \mathrm{nt}$ in size. tmRNAs are found in all bacteria and are highly expressed. The tmRNA has properties of both a tRNA and a mRNA. It participates in trans-translation, which is a reaction that transfers the translational complex to the tmRNA and ultimately leads to degradation of the polypeptide (for review, see [32]).

Based on the size of RNA \#2, we presumed it was the 5S rRNA (114 nt). This was confirmed after cDNA sequencing. Next, RNA \#1 was identified as the $4.5 \mathrm{~S}$ RNA component of the signal recognition particle (FTL_R0044; 108 nt). FTL_R0044 was found in one of ten clones sequenced, whereas the other nine clones were the $5 \mathrm{~S}$ rRNA. This is not very surprising considering the very similar size of these two transcripts and the high expression of the 5S RNA. Despite several attempts, we did not obtain any cDNA clones of RNA $\# 3$, the RNA with an apparent size of $\sim 200$ nt.

\section{Cloning and identification of additional sRNAs}

As our analysis of highly expressed RNA did not result in the identification of novel small RNA transcripts, we proceeded to construct a limited cDNA library of low molecular weight RNAs. For this, we used the same approach as described for the highly expressed sRNAs. Total RNA from bacteria grown under the different conditions described above, but depleted of ribosomal RNAs (16S and 23S rRNA), was separated on a $8 \%$ 


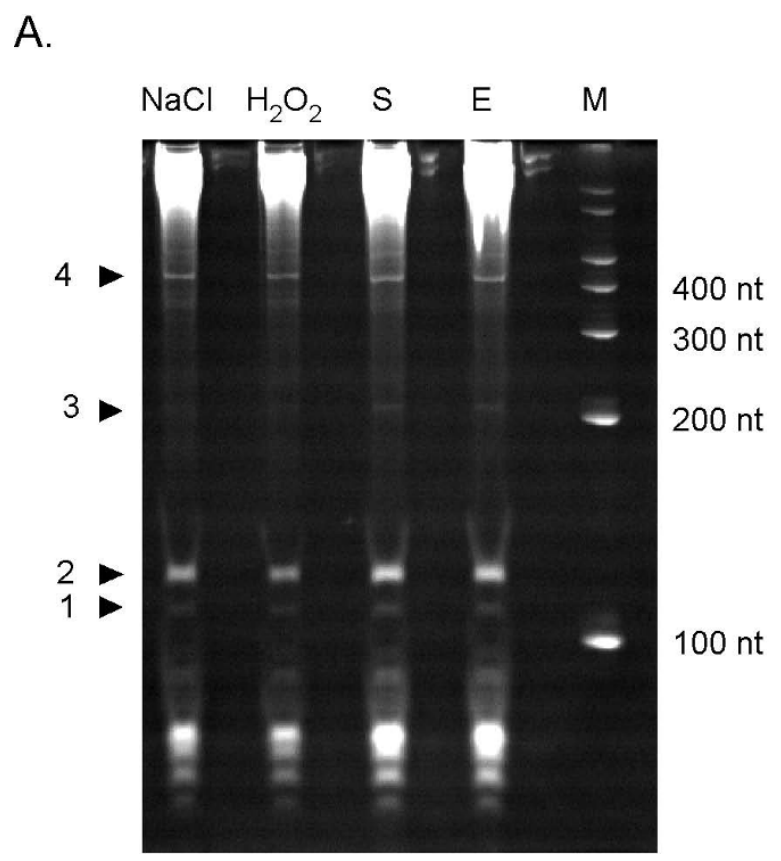

B.

Figure 1 Experimental identification of $\boldsymbol{F}$. tularensis sRNAs. (A) Total RNA extracted at exponential phase (E), stationary phase (S), high salt concentration $(\mathrm{NaCl})$ or after exposure to oxidative stress $\left(\mathrm{H}_{2} \mathrm{O}_{2}\right)$ analyzed by $8 \%$ PAGE and stained by ethidium bromide ( $\mathrm{M}$, RNA marker). Individual bands processed for cloning are indicated by numbers. (B) Schematic representation of gel used for cloning of cDNA. Each square denotes a piece that was individually processed for RNA extraction and A and B designate the area from which FtrA and B were identified, respectively. For clarification, the highly expressed RNAs (\#1-3) seen in (A) are indicated.

denaturing polyacrylamide gel and RNAs of approximately 100-200 nt were eluted from the gel and processed the same way as for the highly expressed RNAs. The gel was divided into smaller areas before eluting the RNAs and ten clones from each area (shown in Figure 1B) were sequenced in order to identify new sRNA species. By this method, we identified two different RNAs, one of which was identified twice from the same gel piece, whereas the remaining clones contained rRNA, tRNA or other contaminating sequences.

To further confirm that the two identified candidates are authentic sRNAs, we performed Northern blotting analysis (Figure 2) using ${ }^{32} \mathrm{P}$-labeled oligonucleotides as probes. As can be seen in Figure 2, a strong band was observed for each of the sRNA candidates with sizes of approximately $\sim 70 \mathrm{nt}$ and $\sim 110 \mathrm{nt}$, respectively. We refer to these sRNAs as FtrA and FtrB for Francisella tularensis sRNA A and B. In addition to the major bands, two bands of larger size were seen for FtrA and one with a lower size for FtrB, suggesting that processing of the transcripts might be occurring.

\section{sRNA mapping, localization, and structure analysis}

To map the ends of the RNAs we performed 5'- and 3'-RACE (Table 1). This revealed that FtrA is $111 \mathrm{nt}$ and FtrB is 115 nt in length. The size found for FtrB is in
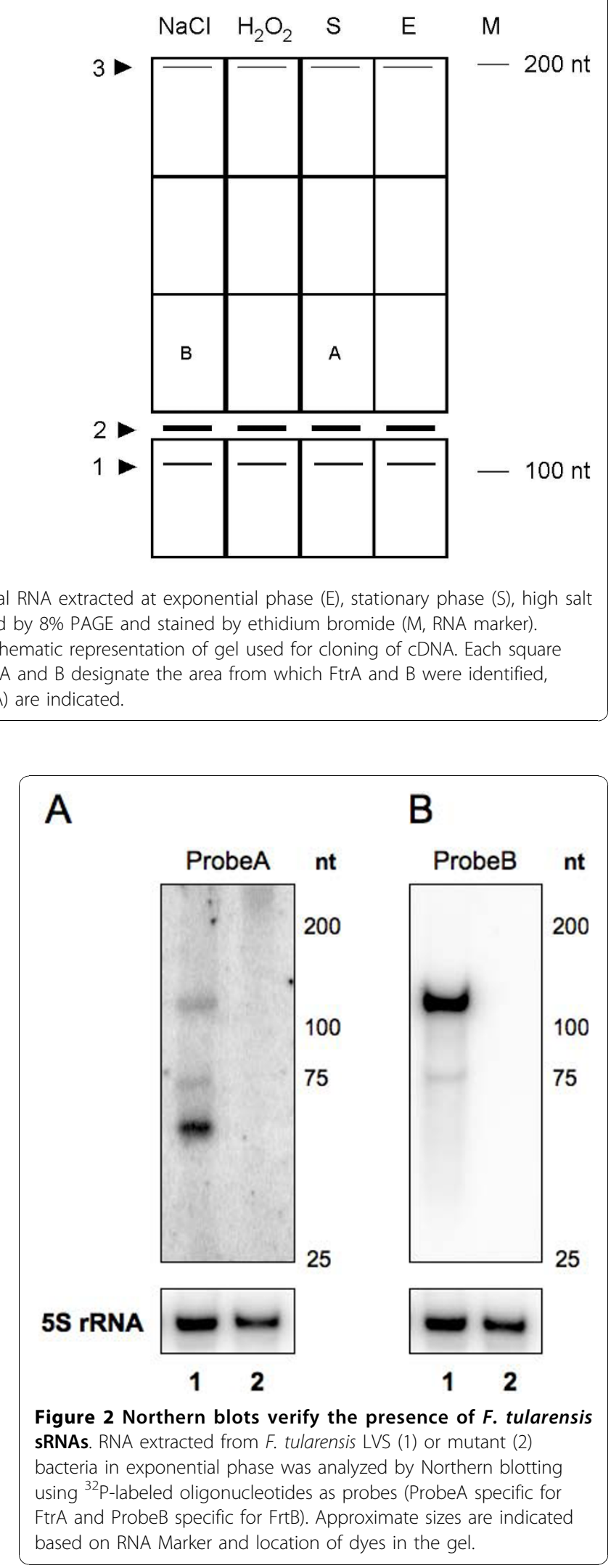
Table 1 Small RNAs identified by cDNA cloning

\begin{tabular}{|c|c|c|c|c|c|}
\hline sRNA & $5^{\prime}$ end $^{a}$ & $3^{\prime}$ end $^{b}$ & $\begin{array}{c}\text { Length } \\
\text { (nt) }\end{array}$ & $\begin{array}{l}\text { Flanking } \\
\text { genes }\end{array}$ & $\begin{array}{l}\text { Genomic } \\
\text { context }^{c}\end{array}$ \\
\hline FtrA & 1256577 & 1256687 & 111 & $\begin{array}{l}\text { FTL_1319- } \\
\text { FTL_1320 }\end{array}$ & $>><$ \\
\hline FtrB & 37655 & 37769 & 115 & $\begin{array}{c}\text { FTL_0035- } \\
\text { FTL_0036 }\end{array}$ & $>>>$ \\
\hline
\end{tabular}

a Coordinate of the $5^{\prime}$ end of sRNA (determined by $5^{\prime}$-RACE).

${ }^{b}$ Coordinate of the 3 ' end of sRNA (determined by 3 '-RACE).

c The orientation of the sRNA and its flanking genes (in order of gene numbers). " $>$ " designates a gene encoded on the coding strand and " $<$ " designates a gene encoded on the non-coding strand.

agreement with the strong bands seen in the Northern blot, whereas it seems to correspond to one of the larger, but less intense, bands seen in the Northern blot of FtrA.

The $\operatorname{ftr} A$ gene is located between FTL_1319 and FTL_1320 (Table 1 and Figure 3) and is encoded on the coding strand. The flanking genes encode a transposase and a conserved hypothetical protein. FtrB is encoded on the coding strand between FTL_0035 and FTL_0036, encoding two hypothetical proteins. The predicted secondary structures of FtrA and FtrB, using the Quikfold program http://dinamelt.bioinfo.rpi.edu/quikfold.php[33,34], are shown in Figure 3. Both FtrA and FtrB are predicted to be highly structured and contain several stem-loops.

To determine whether FtrA and FtrB are similar to known bacterial sRNAs, we performed BLAST to search the sequences of the non-coding RNA database http:// ncrnadb.trna.ibch.poznan.pl/blast.html. No bacterial RNAs in the database showed sequence similarity to the two F. tularensis sRNAs. Sequences were also queried against the Rfam database http://rfam.sanger.ac.uk/, which did not result in any matches. Furthermore, a BLASTN search of the genes encoding either sRNA against the NCBI total sequence database showed that the genes are only found in Francisella and not in any other bacterial genome. This strongly indicates that the two RNAs are novel bacterial sRNAs.

\section{Characterization of ftrA or ftrB mutants}

To study the role of the sRNAs, we proceeded by creating mutant strains carrying a chromosomal deletion of either the $f \operatorname{tr} A$ or $f \operatorname{tr} B$ gene. Sequencing and Northern blotting were respectively used to confirm deletion of these loci and the absence of their corresponding transcripts (Figure 2). Neither mutant strain exhibited any growth defects or increased sensitivity to several stress conditions $\left(\mathrm{H}_{2} \mathrm{O}_{2}\right.$, ethanol, low $\mathrm{pH}, \mathrm{NaCl}$; data not shown), showing that deleting either $\operatorname{ftr} A$ or $\operatorname{ftr} B$ does not affect the ability of $F$. tularensis to survive a number of stresses. Likewise, we did not observe any change in expression of either FtrA or FtrB after exposure to oxidative or osmolarity stress (data not shown).
We next tested if either gene plays a role in F. tularensis intracellular multiplication. As shown in Figure 4, both mutant strains multiplied intracellularly in J774 murine macrophage-like cells in a manner indistinguishable from the wild-type strain, indicating that neither FtrA nor FtrB is required for multiplication in macrophages in vitro.

To examine if FtrA or FtrB is required for virulence of F. tularensis, we assessed the ability of each mutant strain to induce disease in the mouse model of infection. No major difference in survival was observed between mice infected with wild-type LVS or with either of the mutant strains, showing that neither gene is required for virulence (data not shown). In addition, after infecting mice with a 1:1 mixture of mutant and wild-type bacteria we recovered equal numbers of wild-type and mutant bacteria from the spleen (data not shown). This further demonstrates that deleting $f t r A$ or frtB does not affect $F$. tularensis pathogenicity in the mouse model.

It is important to recall that most sRNAs affect gene expression negatively. Deleting a specific sRNA gene and thus alleviating the repressive effect may not be of major consequence for the bacterium. Our results from in vitro characterization of the mutant strains therefore do not rule out the possibility that FtrA or FtrB may indeed control functions related to growth, stress resistance or virulence.

\section{Identification of potential targets}

Most characterized sRNAs control expression of their target genes by base-pairing with a target mRNA. In addition to affecting translation, base-pairing between a sRNA and its target frequently leads to changed stability of both sRNA and mRNA. Therefore, to experimentally identify potential targets for regulation by FtrA and FtrB, we compared the transcriptomes of LVS wild-type bacteria grown in regular broth to that of either the $\operatorname{tr} A$ or the $\operatorname{ftr} B$ mutant. This way we identified several potential targets for FtrA and FtrB (Table 2). None of the potential targets identified by microarray analysis are located in the same genomic region as the sRNAs, indicating that the sRNAs are trans-acting. The analysis identified four genes for which the transcript was found at a higher level in the ftrA mutant than in LVS. These are FTL_0045 (encoding orotidine 5'-phosphate decarboxylase, PyrF), FTL_0207 (encoding pyrrolidone carboxylate peptidase, Pcp), FTL_0902 (encoding an oxidoreductase), and FTL_1922 (encoding a YggT family protein). Interestingly, pyrF transcripts were also found at higher levels in a $h f q$ mutant [30]. Thus, deleting either $h f q$ or $f t r A$ affects pyrF mRNA levels in the same manner, in agreement with a regulatory mechanism in which Hfq-mediated FtrA-pyrF mRNA base-pairing leads to degradation of the target mRNA. This, however, needs to be 


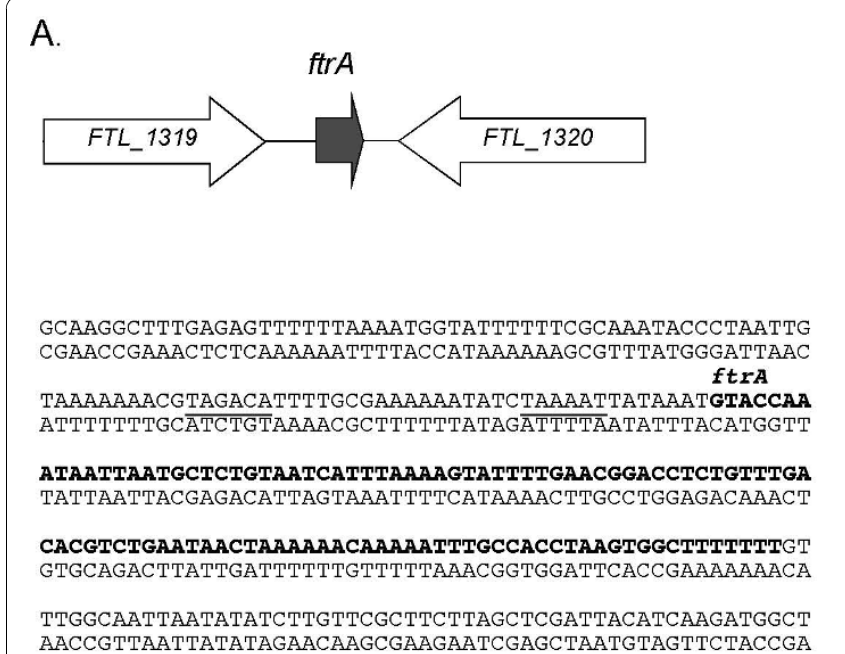

AACCGTTAATTATATAGAACAAGCGAAGAATCGAGCTAATGTAGTTCTACCGA
B.

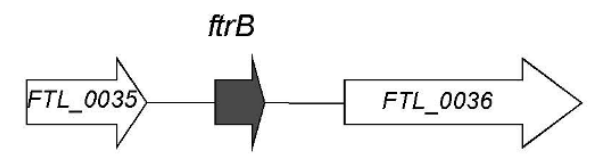

TTTTGAAACTACTAATCGCTAATGTGCTGATTCCCCTTCAAATATTGAAGAGG AAAACTTTGATGATTAGCGATTACACGACTAAGGGGAAGTTTATAACTTCTCC ftrB TGGCTTGCGAAGCAATACGGGGTAGTGTATAAAATTAATATATGATACTAACT
ACCGAACGCTTCGTTATGCCCCATCACATATTTTAATTATATACTATGATTGA

TAACGTCGGTAGTCATTCCCGCGTAGGCGGGATCTCTTTAATTGCTATGAGG ATTGCAGCCATCAGTAAGGGCGCATCCGCCCTTAGAGAAATTAACGATACTCC

TCCCTGTGTCAAGCACGAGGATTGTAAGTTAAATCTCTAGTATAATTTTTACC AGGGACACAGTTCGTGCTCCTAACATTCAATTTAGAGATCATATTAAAAATGG

TAAGGAGTCAAACTAACAAAGGGGCCTGCTTATTAACTAATACTTTCTAGAGC TAACCTCAGTTTGATTGTTTCCCCGGACGAATAATTGATTATGAAAGATCTCG

C.

FtrA

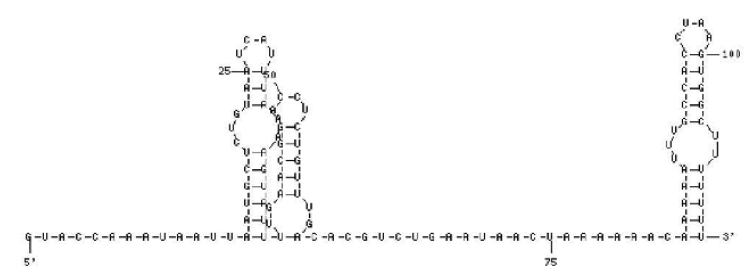

FtrB

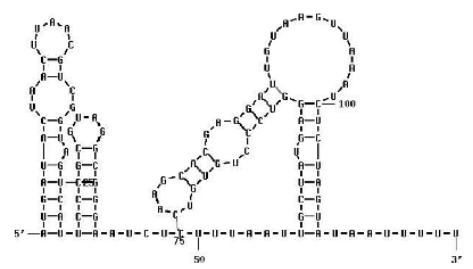

Figure 3 Characterization of the two $\boldsymbol{F}$. tularensis sRNAs. Schematic representations of the genomic positions of the ftrA (A) and ftrB (B) loci are shown at top in each section. The DNA sequence of the regions, with the sRNA gene indicated in bold and proposed -10 and - 35 boxes underlined, are shown below. The secondary structure predictions of FtrA and FtrB using Quikfold (RNA 3.0) are shown in (C).

demonstrated experimentally. The mRNA levels of five genes were changed in the $f t r B$ mutant compared to wild-type bacteria: two were found in higher levels (FTL_0836 and FTL_1754) and three at lower levels

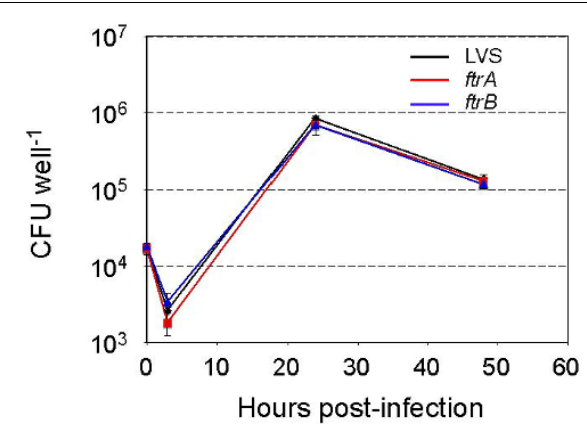

Figure 4 Intracellular macrophage multiplication of sRNA mutants. The number of intracellular bacteria was followed for 48 hours after infection of murine macrophage-like cells $J 774$ by $F$. tularensis LVS wild-type or sRNA mutants ftrA or ftrB.
(FTL_0324, FTL_0421 and FTL_1966) in the ftrB mutant. Of these, FTL_0421 (encoding a lipoprotein) was also found to be down-regulated in the $F$. tularensis $h f q$ mutant [30]. This finding could be explained by a mechanism in which RNA duplex formation leads to stabilization of the mRNA. To confirm the results obtained by microarray analysis, we chose two genes with changed expression in either mutant strain and performed quantitative RT-PCR analysis. For each mutant strain, this analysis confirmed the change in expression for both genes (see Additional File 1). Presently we do not know if FtrA and FtrB regulate these targets directly as it is also possible that the observed changes in mRNA level are due to indirect effects of deleting the sRNA.

\section{Identification of putative sRNAs using bioinformatics analysis}

While our cloning based screen proved effective in identifying previously unknown sRNAs, the relatively small number of clones sequenced meant that only abundant 
Table 2 Effect of ftrA and ftrB deletion on the transcriptome of LVS

\begin{tabular}{|c|c|c|c|}
\hline Locus $^{\mathrm{a}}$ & Gene product & Fold-change in $f t r A^{b}$ & Fold change in $f t r B^{b}$ \\
\hline FTL_0045 & orotidine 5'-phosphate decarboxylase & 1.8 & NA \\
\hline FTL_0207 & pyrrolidone-carboxylate peptidase & 2.6 & NA \\
\hline FTL_0902 & oxidoreductase & 1.8 & NA \\
\hline FTL_1922 & YggT family protein & 10.8 & NA \\
\hline FTL_0324 & pseudogene & NA & 0.7 \\
\hline FTL_0421 & lipoprotein & NA & 0.7 \\
\hline FTL_0836 & hypothetical protein & NA & 8.2 \\
\hline FTL_1754 & hypothetical membrane protein & NA & 1.5 \\
\hline FTL_1966 & anthranilate synthase component I & NA & 0.7 \\
\hline
\end{tabular}

a Genes included had $\geq 1.5$ average fold-change in mRNA level (calculated for all spots) and for which each of the five spots for the gene on the microarray had fold-changes $\geq 1.2$

${ }^{\mathrm{b}}$ Change in mRNA level in mutant strain compared to wild-type LVS

small transcripts could be found using this approach. In an effort to identify putative sRNAs in F. tularensis that may have been missed in our physical screen, we used the bioinformatic tool SIPHT [35] to conduct a genome-wide bioinfomatic screen for candidate sRNAs in F. tularensis LVS. SIPHT identifies candidate intergenic loci based on the co-localization of intergenic conservation and Rho-independent terminators. It then annotates these candidates for several features, including the similarity of their primary sequence and predicted secondary structure to intergenic sequences in other bacterial replicons, their proximity to the putative binding sites of various transcription factors, and their homology to previously confirmed or predicted sRNAs and cis-regulatory RNA elements.

A total of 24 candidate loci were identified by SIPHT, including three intergenic regions (IGRs) each containing two overlapping candidate loci, leading to a total of 21 IGRs (Table 3 and Additional File 2). Importantly, both the FtrA and FtrB sRNA were identified in the bioinformatic prediction (Table 3, candidate 19 (FtrA) and candidate $14(\mathrm{FtrB})$ ), suggesting that other candidate loci identified by SIPHT may represent real non-coding RNAs. One of these candidates was annotated by SIPHT as sharing sequence homology with SprB, a previously identified but uncharacterized sRNA in Staphylococcus aureus [31], while another was found in a similar genomic location based on its flanking genes to P26, an sRNA of unknown function in Pseudomonas aeruginosa. For fourteen of the loci identified, including FtrA and FtrB, conservation of secondary structure was predicted by QRNA [36]. Putative sigma-70 promoters were identified within 400 nucleotides upstream of the predicted terminators of 9 candidates, including two upstream of $f t r A$, one of which in a position consistent with the larger band seen by Northern analysis and the results of the 5'-RACE.

Neither tmRNA nor 4.5S RNA were identified by SIPHT. Further analysis revealed that these loci were missed because they were not associated with predicted Rho-independent terminators. This may reflect a true paucity of Rho-independent terminators in F. tularensis or limitations in the ability of the three terminator predicting algorithms used by SIPHT to identify terminators in an AT rich genomes such as that of $F$. tularensis. The fact that these two sRNAs were not identified by SIPHT suggests that other F. tularensis sRNAs were likely missed in our screen.

Several characteristics of Francisella likely hinder predictions of sRNAs based on the proximity of intergenic sequence conservation and Rho-independent terminators. First, no genome sequences for close relatives of F. tularensis are available for BLAST comparisons and the relatively low GC content (32\%) of the F. tularensis genome leads to spurious hits in BLAST searches that are more likely due to low sequence complexity than to actual sequence conservation. Second, as described above, it is also likely that existing terminator prediction programs, most of which had been trained on E. coli, are less effective in identifying terminators in AT-rich species such as Francisella. Indeed, in a kingdom-wide search for putative sRNAs in over 500 different strains, all Francisella sp. were among the 20 strains with the lowest density of putative intergenic Rho-independent terminators. However, while these factors likely contribute to both the decreased sensitivity and specificity of our sRNA predictions, the fact that both FtrA and FtrB were identified in our in silico screen suggests that the predictive algorithm used by SIPHT is effective in identifying novel sRNAs even in species such as Francisella that are AT-rich and have relatively few sequenced relatives.

Recently, a number of small ORFs encoding small peptides (<50 amino acids), previously non-annotated, have been identified in other bacterial species $[37,38]$. We therefore examined whether the predicted sRNA loci in F. tularensis contain ORFs encoding small peptides (Table 3). A few loci could encode small peptides 
Table 3 Putative sRNAs predicted in silico ${ }^{a}$

\begin{tabular}{|c|c|c|c|c|c|}
\hline RNA & Length (nt) & Coordinates & Flanking genes & Genomic context $^{b}$ & Potential ORFs ${ }^{c}$ \\
\hline 1 & 39 & 599871-599910 & FTL_0609-FTL_0610 & $>68|>| 984<$ & - \\
\hline 2 & 91 & $865504-865595$ & FTL_0886-FTL_0887 & $<1|>| 129>$ & 1 \\
\hline 3 & 168 & 1039023-1039191 & FTL_1090-FTL_1091 & $<4|>|-32>$ & $8,12^{*}$ \\
\hline 4 & 141 & $1567719-1567860$ & FTL_1636-FTL_1637 & $>557|>| 488<$ & $2^{*}$ \\
\hline 5 & 41 & $1808414-1808455$ & FTL_1875-FTL_1876 & $<349|<| 203<$ & $2,8^{*}$ \\
\hline 6 & 89 & $1251973-1252062$ & FTL_1313-FTL_1314 & $>145|<| 47<$ & - \\
\hline 7 & 181 & $1240899-1241080$ & FTL_1303-FTL_1304 & $>26|<| 0<$ & 41 \\
\hline 8 & 125 & $765395-765520$ & FTL_0777-FTL_0778 & $>132|<| 0\rangle$ & 2 \\
\hline 9 & 87 & 508319-508406 & FTL_0527-FTL_0528 & $>149|<| 0>$ & - \\
\hline 10 & 225 & $508181-508406$ & FTL_0527-FTL_0528 & $>11|<| 0\rangle$ & $52^{*}, 32,37$ \\
\hline 11 & 191 & $361680-361871$ & FTL_0391-FTL_0392 & $<476|<| 331<$ & - \\
\hline 12 & 229 & 133934-134163 & FTL_0131-FTL_0132 & $<141|<| 29<$ & 3 \\
\hline 13 & 92 & $52224-52316$ & FTL_0050-FTL_0051 & $<54|<| 0<$ & $7^{*}, 2$ \\
\hline 14 & 177 & $37528-37705$ & FTL_0035-FTL_0036 & $>1|>| 209>$ & $10^{*}, 4,24^{*}, 2$ \\
\hline 15 & 122 & $359671-359793$ & FTL_0389-FTL_0390 & $>1|>| 176<$ & $19,2,2$ \\
\hline 16 & 390 & $511941-512331$ & FTL_0529-FTL_0530 & $>1|>|-32<$ & $9^{*}, 4^{*}, 7,41,7,16^{*}$ \\
\hline 17 & 52 & $527019-527071$ & FTL_R0021-FTL_0544 & $<2|>| 8<$ & - \\
\hline 18 & 36 & $1251854-1251890$ & FTL_1313-FTL_1314 & $>26|<| 219<$ & - \\
\hline 19 & 196 & $1256490-1256686$ & FTL_1319-FTL_1320 & $>57|>| 128<$ & $8,9,13$ \\
\hline 20 & 213 & 1351294-1351507 & FTL_1420-FTL_1421 & $>67|<| 0<$ & $11,19,24^{*}$ \\
\hline 21 & 119 & $1681442-1681561$ & FTL_1744-FTL_1745 & $<33|<| 0<$ & $15^{*}, 3^{*}$ \\
\hline 22 & 292 & $1229654-1229946$ & FTL_R0033-FTL_1289 & $>2|>| 462<$ & $10,13^{*}, 2,11$ \\
\hline 23 & 228 & $49421-49649$ & FTL_0046-FTL_0047 & $>80|<| 49<$ & $2^{*}, 11,12^{*}, 20$ \\
\hline 24 & 240 & $765280-765520$ & FTL_0777-FTL_0778 & $>17|<| 0>$ & $12^{*}, 2,33^{*}$ \\
\hline
\end{tabular}

a sRNA candidates, 5' and 3' ends predicted using SIPHT [35].

b The orientation of the sRNA and its flanking genes (in order of gene numbers). " $>$ " designates a gene encoded on the coding strand and " $<$ " designates a gene encoded on the non-coding strand. Numbers outside of the " $\mid$ " characters denote the distance between the boundaries of the predicted locus and its flanking genes.

' Potential ORFs are indicated by their length in amino acids. ${ }^{*}$ denotes an ORF staring with an alternative start codon.

For sequence of each RNA see Additional File 2.

of 24-52 amino acids, but most loci contained none or only very short ORFs, further supporting the hypothesis that the majority of the loci encode putative RNAs.

\section{Conclusions}

Here, we have confirmed the expression in $F$. tularensis of the well-known non-coding RNAs tmRNA and 4.5S RNA after direct purification and cloning of the cDNAs. Two additional sRNAs expressed by $F$. tularensis were identified from a cDNA library prepared from low molecular weight RNA. The two putative sRNAs (called FtrA and $\mathrm{FtrB}$ ), which are encoded in intergenic regions, were confirmed to be genuine sRNAs by Northern blot analysis and the transcripts were characterized with respect to transcription endpoints. The sRNAs do not show sequence similarity to any known sRNAs and their DNA sequences are only found in Francisella species, indicating that these are novel sRNAs. Deletion of either the $f \operatorname{tr} A$ or $f \operatorname{tr} B$ gene had no effect on bacterial survival during normal growth or stress, and did not affect the capacity of Francisella to induce disease in mice. This suggests that these sRNAs affect functions that are not required under the conditions tested or that additional sRNAs with redundant functions may exist. Specifically, in silico analysis identified conserved sequence between FtrB and sRNA candidate \#20 (Table 3) suggesting they may be functional paralogues. Subsequently, microarray analysis allowed us to identify a number of potential targets of FtrA and FtrB regulation. Further experiments will be needed to verify if these represent real targets. Finally, in silico prediction of putative sRNA loci identified a number of Francisella IGRs, including the two harboring $f t r A$ and $f \operatorname{tr} B$. Future work will determine which of these IGRs indeed encode authentic sRNAs.

\section{Methods}

\section{Bacterial growth and plasmids}

All strains and plasmids used in this study are listed in Table 4. F. tularensis LVS was grown in Schaedler broth supplemented with vitamin K3 (Biomérieux, France), on chocolate agar plates supplemented with IsoVitalex vitamin solution (Biomérieux, France), or in defined 


\begin{tabular}{|c|c|c|c|}
\hline \multicolumn{2}{|l|}{ Strain or plasmid } & \multirow[t]{2}{*}{ Description } & \multirow[t]{2}{*}{ Source or reference } \\
\hline Strain & & & \\
\hline \multicolumn{4}{|l|}{ F. tularensis } \\
\hline & LVS & subsp. holarctica, live vaccine strain & A. Sjöstedt \\
\hline & LVS $\triangle \mathrm{ftrA}$ & LVS with deletion of ftrA & This study \\
\hline & LVS $\triangle \mathrm{ftr} B$ & LVS with deletion of $f t r B$ & This study \\
\hline \multicolumn{4}{|l|}{ E. coli } \\
\hline & $\mathrm{DH} 5 \alpha$ & & Strain collection \\
\hline \multicolumn{4}{|l|}{ Plasmid } \\
\hline & pCR2.1-TOPO & PCR cloning vector, $\mathrm{Km}^{\mathrm{R}}, \mathrm{Amp}^{\mathrm{R}}$ & Invitrogen \\
\hline & pMP812 & $s a c B$ suicide vector, $\mathrm{Km}^{\mathrm{R}}$ & {$[40]$} \\
\hline & pMP- $\triangle$ ftrA & pMP812 containing $\sim 2 \mathrm{~kb}$ fragment for deletion of ftr $\mathrm{A}$ & This study \\
\hline & $\mathrm{pMP}-\triangle \mathrm{ftr} B$ & pMP812 containing $\sim 2 \mathrm{~kb}$ fragment for deletion of $\mathrm{ftr} B$ & This study \\
\hline
\end{tabular}

medium [39]. Escherichia coli was grown in LB. When needed, media was supplemented with kanamycin (50 $\mu \mathrm{g} \mathrm{ml}^{-1}$ for E. coli and $10 \mu \mathrm{g} \mathrm{ml}^{-1}$ for F. tularensis). Oligonucleotides used are described in Table 5.

\section{Bioinformatic prediction of sRNA loci}

Candidate sRNAs were predicted using the SIPHT program, as described previously [35]. Thresholds for BLAST E value, RNAMotif score, FindTerm score, and TransTerm confidence were set to $5 \mathrm{e}-3,-6,-10$, and 86 , respectively. All other parameters were set to default values.

\section{Extraction of RNA for gel fractionation}

LVS was grown to mid-exponential phase or to stationary phase in Schaedler-K3 before harvesting the bacteria. For oxidative stress experiments, bacteria at $\mathrm{OD}_{600}=0.3$ were exposed to $10 \mathrm{mM} \mathrm{H} \mathrm{H}_{2}$ for 30 min before harvested. For experiments at high osmolarity, LVS was grown in complex medium containing $2 \% \mathrm{NaCl}$ to $\mathrm{OD}_{600}=0.3$ before being harvested. Total RNA was extracted using Trizol reagent (Invitrogen) according to the protocol provided by the manufacturer and treated with Turbo DNase (Ambion). For cDNA library construction, total RNA was depleted of $16 \mathrm{~S}$ and $23 \mathrm{~S}$ rRNAs using the MICROBExpress kit (Ambion) according to the protocol provided by the manufacturer before loaded on gel.

\section{RNA elution and CDNA cloning}

$20 \mu \mathrm{g}$ of total RNA was heated and loaded onto an $8 \%$ polyacrylamide/8M urea gel with the RNA Century Plus (Ambion) as size marker. After separation, the gel was stained with ethidium bromide and a gel piece containing RNA of a certain size was cut out. RNA was eluted from the gel after crushing and incubation at $37^{\circ} \mathrm{C}$ in
$0.5 \mathrm{M}$ ammonium acetate and $1 \mathrm{mM}$ EDTA (pH 8.0) for 6 hours under constant agitation. Supernatants were extracted once with chloroform before precipitation of the RNA with isopropanol in the presence of glycogen. Next, 3' adapter was ligated to the dissolved RNA with T4 RNA ligase in the presence of DMSO and RNase inhibitor for $90 \mathrm{~min}$ at $37^{\circ} \mathrm{C}$. The RNA was purified from a $15 \%$ polyacrylamide/urea gel as described above to remove non-incorporated adapters. The eluted RNA was treated with tobacco acid phosphatase (TAP) before ligation of 5' adapter (using the same method as for 3' adapters) and subsequently non-incorporated adapters removed by passing and twice washing the RNA on a Microcon YM-30 (Millipore) column. The RNA was reverse transcribed using the 3'PRIMER and SuperScript II reverse transcriptase (Invitrogen), PCR amplified using 5'PRIMER and 3'PRIMER and ligated into pCR2.1-TOPO. All RNAs were identified using this protocol, except the tmRNA for which we used a protocol that ligates the 3'adapter before the 5 'adapter and utilizes different adapters (adapters B; see Table 5).

\section{Northern blot}

Total RNA was extracted using Trizol according to the manufacturer's instructions (Invitrogen) and quantified on a NanoDrop ND-1000 Spectrophotometer (Thermo Scientific). After extraction, $10 \mu \mathrm{g}$ of total RNA were loaded on a polyacrylamide gel ( $8 \%$ acrylamide/8M urea). After migration, the RNA was transferred to a Hybond-N+ membrane (Amersham) and crosslinked with UV light. The membrane was prehybridized in Rapid-Hyb Buffer (Amersham). Then, ${ }^{32}$ P-labelled genespecific probe (oligonucleotide, see Table 5) was added directly to the prehybridization buffer with the membrane and incubated for 16 hours at $42^{\circ} \mathrm{C}$. After hybridization, the membrane was washed twice with $2 \times \mathrm{SSC} /$ 
Table 5 Oligonucleotides used in this study

\begin{tabular}{|c|c|c|}
\hline Oligonucleotide & Sequence $\left(5^{\prime} \text { to } 3^{\prime} \text { direction }\right)^{a}$ & Use \\
\hline 3' adapter & P-r̈UrUrUCGGGCCGCGGACTGTidT & cDNA cloning and RACE \\
\hline $5^{\prime}$ adapter & GATATGCGCGAATTCCTGTAGAACGAACACTAGGGGrArArA & CDNA cloning and RACE \\
\hline 5'PRIMER & GTAGAACGAACACTAGGGGAAA & CDNA cloning and RACE \\
\hline 3'PRIMER & GACAGTCCGCGGCCCGAAA & cDNA cloning and RACE \\
\hline $3^{\prime}$ adapter_B & P-rUrUrUCTATCCATGGACTGTidT & cDNA cloning tmRNA \\
\hline 5' adapter_B & GATATGCGCGAATTCCTGTAGAACGAACACTAGAAGrArArA & cDNA cloning tmRNA \\
\hline 5'PRIMER_B & GTAGAACGAACACTAGAAGAAA & cDNA cloning tmRNA \\
\hline 3'PRIMER_B & TACAGTCCATGGATAGAAA & cDNA cloning tmRNA \\
\hline ftrA_GSP_5'RACE & GTTATTCAGACGTGTCAAACAGAG & 5'-RACE ftrA \\
\hline ftrA_GSP_3'RACE & GTACCAAATAATTAATGCTCTGTAATC & $3^{\prime}-$ RACE ftrA \\
\hline ftrB_GSP_5'RACE & GAGATTCCCGCCTACGCGG & 5'-RACE ftrB \\
\hline ftrB_GSP_3'RACE & GATACTAACTTAACGTCGGTAGTC & $3^{\prime}$ RACE ftrB \\
\hline ftrA_DelR & GCGCGGCCGCGTGGTAAAATCATCTAGGTTCTAGC & Deletion ftrA \\
\hline ftrA_DelB & CATTTATAATTTAAGATATTITTCGC & Deletion ftrA \\
\hline ftrA_DelO & GCGAAAAAATATCTAAAATTATAAATGGGCAATTAATATATCTTGTTCGCTTCTTAGC & Deletion ftrA \\
\hline ftrA_DelT & GCGTCGACGCAACTAAGAAAAGAATATTTAATAGCC & Deletion ftrA \\
\hline ftrA_DelCheck1 & CATATGTAGTGTACTTTATTTAAATAC & Verification of ftrA deletion \\
\hline ftrA_DelCheck2 & CCTAAGTTTCAGTTGCTGAATTATTTGG & Verification of ftrA deletion \\
\hline ftrA_DelCheck3 & GCCACTGAAGGCGGAAATCTCGC & Verification of ftrA deletion \\
\hline ftrA_DelCheck4 & CAGTTAAATATTATTAACATTAAGAAAC & Verification of ftrA deletion \\
\hline ftrB_DelR & GCGCGGCCGCCTTTTAAGATTTGTATTCTTATTTGTTC & Deletion $\mathrm{ftrB}$ \\
\hline ftrB_DelB & CACTACCCCGTATTGCTTCGCAAGCC & Deletion ftrB \\
\hline ftrB_DelO & GGCTTGCGAAGCAATACGGGGTAGTGCCTAAGGAGTCAAACTAACAAAGGGGCCTGC & Deletion $\mathrm{ftr} B$ \\
\hline ftrB_DelT & GCGTCGACCAGAGCATTTATGATAGTTTGTTTCC & Deletion ftrB \\
\hline ftrB_DelCheck1 & CTAAATCTAAGGAATGATAATTAACC & Verification of ftrB deletion \\
\hline ftrB_DelCheck2 & GGACAGGAATGGACAGCAGAAG & Verification of ftrB deletion \\
\hline ftrB_DelCheck3 & GTATATCCTATTTGAAAAGCTAATGGC & Verification of ftrB deletion \\
\hline ftrB_DelCheck4 & CACTATATGGATATGCTTATGAACAAGC & Verification of ftrB deletion \\
\hline ProbeA & CAGACGTGTCAAACAGAGGTCCGTTCAAAATAC & Northern blot \\
\hline ProbeB & GAGATTCCCGCCTACGCGGGAATGACTACCGACG & Northern blot \\
\hline FTL_0044_F & GCTATATGTCCCAGGTGTAAGG & qRT-PCR \\
\hline FTL_0044_R & GCTCTTTGGCTITITTAGGGGTC & qRT-PCR \\
\hline FTL_0421_F & GGGCAACTGTAACAGTTAAGC & qRT-PCR \\
\hline FTL_0421_R & CTTCTITGTCATAAACTACATTAGC & qRT-PCR \\
\hline FTL_0836_F & GTGGCTATTGATGACATACTCAAC & qRT-PCR \\
\hline FTL_0836_R & GCTAAGCCTAGATAACTGATACC & qRT-PCR \\
\hline FTL_1922_F & GGATTTGATTITCTCCAATTATTG & qRT-PCR \\
\hline FTL_1922_R & CTGCGCAATAATGCTITGTATG & qRT-PCR \\
\hline
\end{tabular}

${ }^{a}$ Bases preceded by $r$ designates a ribonucleotide whereas all other are deoxyribonucleotides. idT designates an inverted deoxythymidine. $\mathrm{P}$ designates a phosphorylated $5^{\prime}$ end. Notl site is underlined. Sall site is shown in italics.

$0.1 \%$ SDS, once with $1 \times \mathrm{SSC} / 0.1 \%$ SDS and twice with $0.1 \times$ SSC/0.1\% SDS. Results were analyzed on a Storm 860 PhosphorImager (Molecular Dynamics) using the ImageQuant software (Molecular Dynamics).

\section{5'- and 3'-RACE}

For 5'-RACE, $15 \mu \mathrm{g}$ of total RNA was incubated with TAP for 2 hours at $37^{\circ} \mathrm{C}$ after which $5^{\prime}$ adapter was ligated with RNA ligase. RNA was purified from gel and reverse transcribed with a gene specific primer and
SuperScript II RT. cDNA was then used as a template in PCR reaction with the 5'PRIMER and the gene specific primer (GSP_5'RACE) and cloned into pCR2.1TOPO before sequencing.

3'-RACE was performed by ligating the 3' adapter to total RNA, followed by gel purification of adapter ligated RNA, reverse transcription with 3'PRIMER and finally conducting PCR with 3'PRIMER and GSP_3'RACE. The 3 ' end of each sRNA was determined by sequencing of cloned PCR products in PCR2.1-TOPO. 


\section{Construction of ftrA and ftrB mutants}

Regions of approximately $1 \mathrm{~kb}$ upstream and downstream of $f t r A$ and $f t r B$ were amplified by PCR using primer pairs DelR/DelB and DelO/DelT. The upstream and downstream fragments were purified from gel, annealed and extended in 20 cycles of PCR without primers and the product further used as template in a PCR reaction with primers DelR and DelT. The $\sim 2 \mathrm{~kb}$ PCR products were cloned into pCR2.1-TOPO (Invitrogen) and mobilized as a NotI-SacI fragment into the $s a c B$ based suicide vector pMP812 [40] creating pMP- $\Delta$ ftr $A$ and pMP- $\Delta f \operatorname{tr} B$. pMP- $\Delta f \operatorname{tr} A$ and $\mathrm{pMP}-\Delta f \operatorname{tr} B$ were introduced in LVS by electroporation and integration of the plasmid into the chromosome confirmed by PCR. Strains were then passed once in medium without selection, subsequently streaked on solid medium containing $6 \%$ sucrose and isolated colonies were tested for loss of the gene by PCR (using primer pairs DelCheck1/DelCheck4 and DelCheck2/DelCheck3). Deletion of the gene was confirmed by sequencing.

\section{cDNA labeling and microarray hybridizations}

RNA used in microarray experiments was extracted using Trizol reagent combined with purification of the aqueous phase on RNeasy columns (Qiagen). cDNA labeling and microarray hybridizations were performed as described [30]. Three independent experiments and RNA extractions were performed and each set of RNAs was used in one hybridization experiment. The F. tularensis microarrays (obtained from the "Pathogen Functional Genomics Resource Center", PFGRC) contain 70-mer oligonucleotides, in five copies, representing all genes of strains SchuS4 and LVS. Microarrays were scanned with a Genepix 4000B scanner (Molecular Devices). To quantify signal fluorescence intensities, TIFF images were analyzed using the Genepix Pro 6.0 software. Statistical analyses were performed using publicly available software, the R/Bioconductor package LIMMA (available from http://www.bioconductor.org). A list of statistically significant differentially expressed genes was obtained using lowess normalization (after inspection of MA plots) and applying the empirical Bayes moderated t-test. Microarray data are available at ArrayExpress.

\section{Quantitative real-time RT-PCR}

To validate the microarray results, two ORFs were selected (for each mutant strain) for real-time quantitative reverse transcription PCR (qRT-PCR) analysis with the same RNA samples used in the microarray hybridizations. For the analyses, one microgram of RNA was reverse transcribed using random hexamers and SuperScript II reverse transcriptase (Invitrogen) according to the protocol provided by the manufacturer. Real-time
PCR was performed with gene-specific primers using an ABI PRISM 7700 and FastStart SYBR master mix (Roche Diagnostics). To calculate the amount of genespecific transcript, a standard curve was plotted for each primer set using a series of diluted genomic DNA from LVS. To compare the transcript amounts in the different strains, the amounts of each gene transcript was normalized to DNA helicase (FTL_1656) as this gene has been shown to change little in expression during growth [23]. The expression of each gene was determined from three replicates in a single qRT-PCR experiment.

\section{Cell infections}

J774 cells were propagated in RPMI or Dulbecco's Modified Eagle's Medium (DMEM) medium containing 10\% fetal calf serum. Cells were seeded at a concentration of $\sim 2 \times 10^{5}$ cells per well in 12-well tissue plates (Falcon) and monolayers were used 24 hours after seeding. J774 macrophage monolayers were incubated for $90 \mathrm{~min}$ at $37^{\circ} \mathrm{C}$ with the bacterial suspensions (approximate multiplicities of infection 100) to allow the bacteria to enter. After washing (time zero of the kinetic analysis), the cells were incubated in fresh culture medium containing gentamicin $\left(10 \mu \mathrm{g} \mathrm{ml}^{-1}\right)$ to kill extracellular bacteria. At several time-points, cells were washed three times in PBS and processed for counting of surviving intracellular bacteria. For this, bacteria were recovered by lysis of macrophages with distilled water and the titer of viable bacteria released from the cells was determined by spreading preparations on agar plates. For each strain and time in an experiment, the assay was performed in triplicate. Each experiment was independently repeated two times and the data presented are from one experiment.

\section{Mice infections}

LVS and mutant strains were grown in Schaedler-K3 to exponential growth phase and diluted to the appropriate concentrations. 6 to 8-week-old female BALB/c mice (Janvier, Le Genest St Isle, France) were i.p. inoculated with $200 \mu \mathrm{l}$ of bacterial suspension. Groups of five mice were inoculated with various doses of bacteria (approximately $10^{1}$ to $10^{4}$ bacteria) and the mortality was followed for 9 days. The actual number of viable bacteria in the inoculum was determined by plating dilutions of the bacterial suspension on chocolate plates. For competitive infections, LVS and mutant bacteria were mixed in 1:1 ratio and a total of approximately 400 bacteria were used for infection of five mice. After four days, mice were sacrificed. Homogenized spleen tissue from the five mice in one experiment were mixed, diluted and spread onto chocolate agar plates. PCR to distinguish wild-type and mutant bacteria were performed on 100 colonies. Animal experiments were performed according 
to the INSERM guidelines for laboratory animals' husbandry.

\section{Additional material}

Additional file 1: Quantitative RT-PCR confirms the microarray results. Transcript levels of selected genes were normalized to that of DNA helicase (FTL_1656) and the fold difference (in mutant strain relative to wild-type strain) and standard deviations are shown for the FTL_0045 and FTL_1922 genes (ftrA mutant) and FTL_0421 and FTL_0836 genes (ftrB mutant).

Additional file 2: Putative sRNAs predicted in silico. Table contains data provided in Table 3 in the text and the sequences of each of the predicted RNAs.

\section{Acknowledgements}

This work was funded by INSERM, CNRS and Université Paris Descartes. We thank Dr. M. Pavelka for providing plasmid pMP812. DNA microarrays were obtained through NIAID's Pathogen Functional Resource Center, managed and funded by Division of Microbiology and Infectious Diseases, NIAID, NIH, DHHS and operated by The Institute for Genomic Research (TIGR).

\section{Author details}

${ }^{1}$ INSERM U1002, Paris, France. ${ }^{2}$ Université Paris Descartes, Faculté de Médecine Necker-Enfants Malades, Paris, France. ${ }^{3}$ Broad Institute of MIT and Harvard, Cambridge, USA. ${ }^{4}$ Channing Laboratories, Brigham and Women's Hospital, Harvard Medical School, Boston, USA.

\section{Authors' contributions}

GP carried out RNA extraction, cDNA library construction, Northern hybridizations, RACE experiments, GRT-PCR, mutant constructions and mutant characterizations. EF performed microarray hybridizations and analysis. $J \mathrm{~L}$ carried out the bioinformatic SRNA prediction and annotations. MD carried out cell culture studies and ID the animal experiments. AC participated in design of study and writing of manuscript. KLM coordinated research, analyzed data, and wrote manuscript. All authors read and approved the final manuscript.

Received: 19 July 2010 Accepted: 10 November 2010 Published: 10 November 2010

\section{References}

1. Waters LS, Storz G: Regulatory RNAs in bacteria. Cell 2009, 136(4):615-628.

2. Morfeldt E, Taylor D, von Gabain A, Arvidson S: Activation of alpha-toxin translation in Staphylococcus aureus by the trans-encoded antisense RNA, RNAlll. Embo J 1995, 14(18):4569-4577.

3. Geisinger E, Adhikari RP, Jin R, Ross HF, Novick RP: Inhibition of rot translation by RNAlll, a key feature of agr function. Mol Microbiol 2006, 61(4):1038-1048.

4. Boisset $\mathrm{S}$, Geissmann $\mathrm{T}$, Huntzinger $\mathrm{E}$, Fechter $\mathrm{P}$, Bendridi N, Possedko M, Chevalier C, Helfer AC, Benito Y, Jacquier A, et al: Staphylococcus aureus RNAlll coordinately represses the synthesis of virulence factors and the transcription regulator Rot by an antisense mechanism. Genes Dev 2007, 21(11):1353-1366.

5. Novick RP, Ross HF, Projan SJ, Kornblum J, Kreiswirth B, Moghazeh S: Synthesis of staphylococcal virulence factors is controlled by a regulatory RNA molecule. Embo J 1993, 12(10):3967-3975.

6. Huntzinger $E$, Boisset $S$, Saveanu $C$, Benito $Y$, Geissmann T, Namane A, Lina G, Etienne J, Ehresmann B, Ehresmann C, et al: Staphylococcus aureus RNAIII and the endoribonuclease III coordinately regulate spa gene expression. Embo J 2005, 24(4):824-835.

7. Padalon-Brauch G, Hershberg R, Elgrably-Weiss M, Baruch K, Rosenshine I, Margalit H, Altuvia S: Small RNAs encoded within genetic islands of Salmonella typhimurium show host-induced expression and role in virulence. Nucleic Acids Res 2008, 36(6):1913-27.
8. Julio SM, Heithoff DM, Mahan MJ: ssrA (tmRNA) plays a role in Salmonella enterica serovar Typhimurium pathogenesis. J Bacteriol 2000, 182(6):1558-1563.

9. Altier C, Suyemoto M, Ruiz Al, Burnham KD, Maurer R: Characterization of two novel regulatory genes affecting Salmonella invasion gene expression. Mol Microbiol 2000, 35(3):635-646.

10. Santiviago CA, Reynolds MM, Porwollik S, Choi SH, Long F, AndrewsPolymenis HL, McClelland M: Analysis of pools of targeted Salmonella deletion mutants identifies novel genes affecting fitness during competitive infection in mice. PLoS Pathog 2009, 5(7):e1000477.

11. Song T, Mika F, Lindmark B, Liu Z, Schild S, Bishop A, Zhu J, Camilli A, Johansson J, Vogel J, et al: A new Vibrio cholerae sRNA modulates colonization and affects release of outer membrane vesicles. $\mathrm{Mol}$ Microbiol 2008, 70(1):100-111.

12. Lenz DH, Miller MB, Zhu J, Kulkarni RV, Bassler BL: CsrA and three redundant small RNAs regulate quorum sensing in Vibrio cholerae. Mol Microbiol 2005, 58(4):1186-1202.

13. Lenz DH, Mok KC, Lilley BN, Kulkarni RV, Wingreen NS, Bassler BL: The small RNA chaperone Hfq and multiple small RNAs control quorum sensing in Vibrio harveyi and Vibrio cholerae. Cell 2004, 118(1):69-82.

14. Kreikemeyer B, Boyle MD, Buttaro BA, Heinemann M, Podbielski A: Group A streptococcal growth phase-associated virulence factor regulation by a novel operon (Fas) with homologies to two-component-type regulators requires a small RNA molecule. Mol Microbiol 2001, 39(2):392-406.

15. Li Z, Sledjeski DD, Kreikemeyer B, Podbielski A, Boyle MD: Identification of pel, a Streptococcus pyogenes locus that affects both surface and secreted proteins. J Bacteriol 1999, 181(19):6019-6027.

16. Heurlier K, Williams F, Heeb S, Dormond C, Pessi G, Singer D, Camara M, Williams P, Haas D: Positive control of swarming, rhamnolipid synthesis, and lipase production by the posttranscriptional RsmA/RsmZ system in Pseudomonas aeruginosa PAO1. J Bacteriol 2004, 186(10):2936-2945.

17. Ventre I, Goodman AL, Vallet-Gely I, Vasseur P, Soscia C, Molin S, Bleves S, Lazdunski A, Lory S, Filloux A: Multiple sensors control reciprocal expression of Pseudomonas aeruginosa regulatory RNA and virulence genes. Proc Natl Acad Sci USA 2006, 103(1):171-176.

18. Burrowes E, Abbas A, O'Neill A, Adams C, O'Gara F: Characterisation of the regulatory RNA RsmB from Pseudomonas aeruginosa PAO1. Res Microbiol 2005, 156(1):7-16.

19. Bordi C, Lamy MC, Ventre I, Termine E, Hachani A, Fillet S, Roche B, Bleves S, Mejean V, Lazdunski A, et al: Regulatory RNAs and the HptB/RetS signalling pathways fine-tune Pseudomonas aeruginosa pathogenesis. Mol Microbiol 2010, 76(6):1427-43.

20. Grieshaber NA, Grieshaber SS, Fischer ER, Hackstadt T: A small RNA inhibits translation of the histone-like protein $\mathrm{Hc} 1$ in Chlamydia trachomatis. Mol Microbiol 2006, 59(2):541-550.

21. Shimizu T, Yaguchi H, Ohtani K, Banu S, Hayashi H: Clostridial VirR/VirS regulon involves a regulatory RNA molecule for expression of toxins. $\mathrm{Mol}$ Microbiol 2002, 43(1):257-265.

22. Ohtani K, Bhowmik SK, Hayashi H, Shimizu T: Identification of a novel locus that regulates expression of toxin genes in Clostridium perfringens. FEMS Microbiol Lett 2002, 209(1):113-118.

23. Brotcke A, Weiss DS, Kim CC, Chain P, Malfatti S, Garcia E, Monack DM: Identification of MglA-regulated genes reveals novel virulence factors in Francisella tularensis. Infect Immun 2006, 74(12):6642-6655.

24. Lauriano CM, Barker JR, Yoon SS, Nano FE, Arulanandam BP, Hassett DJ, Klose KE: MgIA regulates transcription of virulence factors necessary for Francisella tularensis intraamoebae and intramacrophage survival. Proc Natl Acad Sci USA 2004, 101(12):4246-4249.

25. Charity JC, Costante-Hamm MM, Balon EL, Boyd DH, Rubin EJ, Dove SL: Twin RNA polymerase-associated proteins control virulence gene expression in Francisella tularensis. PLoS Pathog 2007, 3(6):e84.

26. Mohapatra NP, Soni S, Bell BL, Warren R, Ernst RK, Muszynski A, Carlson RW, Gunn JS: Identification of an Orphan Response Regulator Required for the Virulence of Francisella spp. and Transcription of Pathogenicity Island Genes. Infect Immun 2007, 75(7):3305-3314.

27. Brotcke $A$, Monack DM: Identification of fevR, a novel regulator of virulence gene expression in Francisella novicida. Infect Immun 2008, 76(8):3473-3480

28. Charity JC, Blalock LT, Costante-Hamm MM, Kasper DL, Dove SL: Small molecule control of virulence gene expression in Francisella tularensis. PLoS Pathog 2009, 5(10):e1000641. 
29. Grall N, Livny J, Waldor M, Barel M, Charbit A, Meibom KL: Pivotal role of the Francisella tularensis heat-shock sigma factor RpoH. Microbiology 2009, 155(Pt 8):2560-2572.

30. Meibom KL, Forslund AL, Kuoppa K, Alkhuder K, Dubail I, Dupuis M, Forsberg A, Charbit A: Hfq, a novel pleiotropic regulator of virulenceassociated genes in Francisella tularensis. Infect Immun 2009, 77(5):1866-1880

31. Pichon C, Felden B: Small RNA genes expressed from Staphylococcus aureus genomic and pathogenicity islands with specific expression among pathogenic strains. Proc Natl Acad Sci USA 2005, 102(40):14249-14254.

32. Keiler KC: Biology of trans-translation. Annu Rev Microbiol 2008, 62:133-151.

33. Markham NR, Zuker M: DINAMelt web server for nucleic acid melting prediction. Nucleic Acids Res 2005, 33(Web Server issue):W577-581.

34. Zuker M: Mfold web server for nucleic acid folding and hybridization prediction. Nucleic Acids Res 2003, 31(13):3406-3415.

35. Livny J, Teonadi H, Livny M, Waldor MK: High-throughput, kingdom-wide prediction and annotation of bacterial non-coding RNAs. PLOS One 2008, 3(9):e3197.

36. Rivas E, Eddy SR: Noncoding RNA gene detection using comparative sequence analysis. BMC Bioinformatics 2001, 2:8.

37. Hemm MR, Paul BJ, Schneider TD, Storz G, Rudd KE: Small membrane proteins found by comparative genomics and ribosome binding site models. Mol Microbiol 2008, 70(6):1487-1501.

38. Schmalisch M, Maiques E, Nikolov L, Camp AH, Chevreux B, Muffler A, Rodriguez S, Perkins J, Losick R: Small genes under sporulation control in the Bacillus subtilis genome. J Bacteriol 2010, 192(20):5402-5412.

39. Chamberlain RE: Evaluation of Live Tularemia Vaccine Prepared in a Chemically Defined Medium. Appl Microbiol 1965, 13:232-235.

40. LoVullo ED, Molins-Schneekloth CR, Schweizer HP, Pavelka MS: Single-copy chromosomal integration systems for Francisella tularensis. Microbiology 2009, 155(Pt 4):1152-1163.

doi:10.1186/1471-2164-11-625

Cite this article as: Postic et al:: Identification of small RNAs in

Francisella tularensis. BMC Genomics 2010 11:625.

\section{Submit your next manuscript to BioMed Central and take full advantage of:}

- Convenient online submission

- Thorough peer review

- No space constraints or color figure charges

- Immediate publication on acceptance

- Inclusion in PubMed, CAS, Scopus and Google Scholar

- Research which is freely available for redistribution 